\title{
Recent developments concerning the dispersion of carbon nanotubes in surfactant/polymer systems by MD simulation
}

\author{
S. Mahmood Fatemi ${ }^{1} \cdot$ Masumeh Foroutan $^{1}$
}

Received: 7 July 2015/Accepted: 28 August 2015/Published online: 9 November 2015

(c) The Author(s) 2015. This article is published with open access at Springerlink.com

\begin{abstract}
Carbon nanotubes (CNTs) hold the promise of delivering exceptional mechanical properties and multifunctional characteristics due to their unique physiochemical properties and prospective applications in various nanotechnologies. However, current techniques of CNTs fabrication cannot produce homogenous CNTs, and this prevents the widespread use of CNTs. Ever-increasing interest in applying CNTs in many different fields has led to continued efforts to develop dispersion and functionalization techniques. Techniques for separating bundles of CNTs into homogeneous dispersion are still under development. The preparation of effective dispersions of CNTs presents a major impediment to the extension and utilization of CNTs. CNTs intrinsically tend to bundle and/or aggregate. The prevention of such behavior has been explored by testing various techniques to improve the dispersibility of CNTs in a variety of solvents. There are mainly two approaches to obtain a good quality dispersion; chemical functionalization and physical interactions. The chemical functionalization technique has been found effective, but deteriorates the intrinsic properties of CNTs through the introduction of defects in the wall. Physical blending approaches with the ultrasound and high speed shearing have been proven capable of debundling CNTs and stabilizing individual CNTs while maintaining their integrity and intrinsic properties. Contemporary methods for dispersion of CNTs in aqueous media are discussed and most attention is paid to molecular dynamics simulation
\end{abstract}

Masumeh Foroutan

foroutan@ut.ac.ir; foroutan@khayam.ut.ac.ir

1 Department of Physical Chemistry, School of Chemistry, College of Science, University of Tehran, Tehran, Iran techniques and other physical techniques, as well as to the use of various surfactants and polymers.

Keywords Molecular dynamics (MD) simulation · Carbon nanotubes (CNTs) · Dispersion · Polymer · Surfactant

\section{Introduction}

CNTs have been considered as a promising candidate for the next generation high performance. Outstanding mechanical [1], electrical [2], and thermal properties [3] of CNTs makes them a promising candidate for a wide variety of applications, such as biomedical science [4], field emission [5], super-capacitors [6], molecular sensors [7] reinforced composites [8], therapeutics [9], antitumor therapeutics [10], gene or drug delivery [11], transistors [12], solar cells [13], catalyst supports [14], hydrogen storage [15], gas separation [16], lithium batteries [17] and so on $[18,19]$. However, their insolubility in both water and organic solvents hinders the path toward the practical applications of this unique class of materials. Pristine nanotubes tend to assemble in bundles that contain hundreds of close-packed CNTs. It is difficult to prepare stable aqueous dispersions of CNTs; their insolubility has been a limitation for the practical applications of this unique material. Proper dispersion of CNT materials is important to retaining the electronic properties of the nanotubes. Currently, the main approaches used to disperse nanotubes are either mechanical or chemical. The mechanical approach consists of ultra-sonication and high shear mixing. These processes are time-consuming and have low efficiency. They can result in the fragmentation of CNTs and subsequent decrease of their aspect ratio and 
can lead to poor stability of the dispersion [20]. The chemical approach [21, 22] entails both covalent [23] and non-covalent [24] methods and is aimed at improving the chemical compatibility of CNTs with the target medium and at reducing their tendency to agglomerate. Covalent methods involve chemical modification (or chemical functionalization) of the CNT walls with various chemical moieties to improve solubility in solvents [25]. This aggressive approach may introduce defects in the CNT walls and modify the $\pi$-electron conjugation, which may result in the deterioration of their peculiar properties. Noncovalent functionalization, instead, is based on non-covalent interactions such as the physical adsorption of molecules on the CNT surface [26]. It is attractive because the $\pi$-electron cloud of the graphene sheet of the CNT is not disturbed and the characteristic properties of the CNT are preserved. To exfoliate the CNTs bundles, the tube surface can be modified, via van der Waals forces and $\pi-\pi$ interactions, by adsorption or wrapping of surfactants, polymers, or biomolecules. This method will also facilitate the reduction of time and cost required to produce CNT reinforced nanocomposite materials as well as homogeneous CNT dispersed solutions for many applications. Selfassembled structures adsorbed on CNTs offer a plethora of opportunities to endow them with new functions and to integrate them into devices and materials. At the same time they are keys to solve the greatest problem in CNT utilization-debundling and individualization. Several models attempt to describe the phenomenon, but detailed interpretation of the experimental results is often difficult since direct evidence of the structures is not trivial to obtain and different experiments have led to contradictory conclusions. Computer simulations are a convenient tool to study the solvation of CNTs because they can give a microscopic picture of the process. They avoid some of the experimental difficulties associated with the observation of the structures and ultimately afford a theoretical understanding of the effects that play a role in the dispersion of CNTs in solution. Molecular dynamic (MD) simulation can describe in detail the interface and morphology of molecules adsorbed on carbon surfaces and, more importantly, the interaction mechanisms that take place in different supramolecular aggregates [27]. In this review, we present recent developments concerning the dispersion of CNTs in surfactant and polymer systems by MD simulation.

\section{Dispersion of CNTs by surfactant}

Considerable research efforts have already been devoted to optimize and develop processes for dispersion of CNTs. Currently, techniques for separating bundles of CNTs into homogeneous dispersions are still under development, although a few methods have been successful at the laboratory scale. One of the main approaches to disperse and exfoliate CNTs is based on the use of surfactants [28], with their use, tremendous progress has been made to stabilize CNT dispersions. Despite the efficiencies of anionic, cationic, and nonionic surfactants have been demonstrated to different extents, the exact mechanism by which CNTs and the different surfactants interact is still uncertain. Many researchers have suggested that van der Waals interactions, $\pi-\pi$ stacking, and hydrophobic interaction are major factors that account for the CNTs dispersion and non-functionalized CNTs can be solubilized in suitably chosen organic solvents.

Recently Santos et al. [29] have presented MD simulations of the water-surfactant-single walled carbon nanotubes (SWNTs) system. A mixture of two anionic amphiphilic, namely sodium dodecyl sulfate and sodium cholate, presented the best performance in discriminating nanotubes by diameter. The simulations revealed one aspect of the discriminating power of surfactants: they can actually be attracted toward the interior of the nanotube cage. In addition, Striolo et al. [30] have presented results from all-atom MD for aqueous flavin mononucleotide (FMN). They report results for the aggregate morphology of FMN on SWNTs of different diameters, as well as the potential of mean force (PMF) between $(6,6)$ SWNTs in the presence of aqueous FMN. Their simulations indicated that at low SDS coverage, a monolayer form in which SDS molecules orient parallel to the tube surface. Their results indicate that SDS molecules at low coverage on SWNTs are very mobile. At short SWNT-SWNT distance, they tend to accumulate between the interacting tubes because in so doing one SDS molecule interacts with both SWNT surfaces simultaneously. Unfortunately, when this happens, a portion of the SWNT surface remains exposed to water, which may lead to nanotube aggregation. A snapshot is provided in Fig. 1 to illustrate this situation.

In addition, dispersion of CNT with SDS surfactant was reported by Duan et al. [31] via MD simulations from an energy perspective. They showed that four congregation processes were identified to reveal the aggregation morphologies of SDS surfactants on the surface of CNTs as well as the effect of the diameter of a CNT on the adsorption density. Results show that, with the initial gap of $6.5 \AA$, 8 SDS molecules can penetrate and wrap onto the surface of the CNT, as shown in Fig. 2a. They found that 8 SDS molecules are the minimum number to keep the separation distance of $6.5 \AA$ and the two CNTs will approach toward each other if the number of SDS is less than 8 . With 8 SDS molecules, the individual CNTs are not able to re-attach to form a bundle, but have a slight misalignment. As the spacing between two CNTs increases, more SDS molecules attach to the gap. Finally, when 


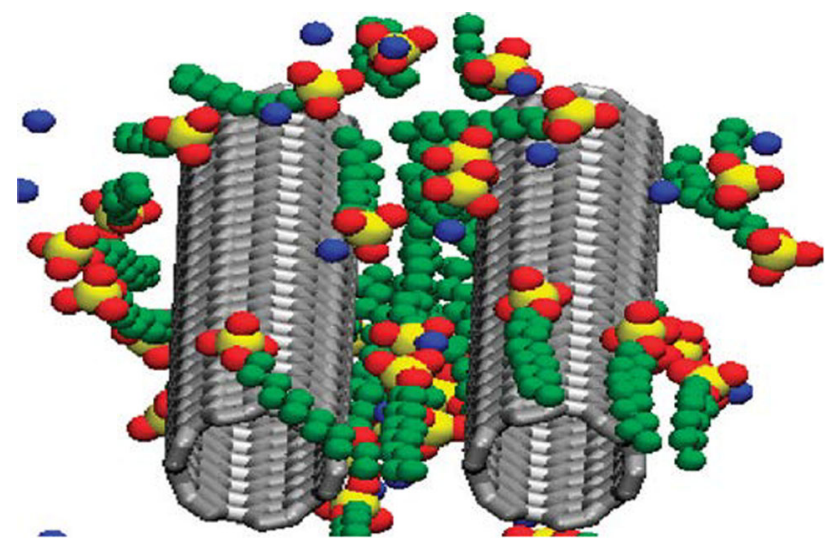

Fig. 1 Representative simulation snapshots for SDS aggregates on two approaching SWNTs at low SDS surface coverage. The SWNTs are separated by $6.90 \AA$ A Green, red, and yellow spheres represent methyl groups, oxygen, and sulfur atoms of SDS, respectively. Blue spheres represent sodium ions. Carbon atoms in nanotubes are connected with bold gray lines. Water is not shown for clarity. Reprinted (adapted) with permission from Ref. [30]. Copyright (2015) American Chemical Society

the gap between two CNTs reaches $10 \AA$, 18 SDS molecules are required to keep the gap open, as shown in Fig. 2b. The two initially parallel CNTs are completely misaligned, with a shortest distance of around $10 \AA$.

To aid elucidate the role of surfactant structure in the CNT dispersion process, Sohrabi et al. [32] have reported the results of fully atomistic MD simulations of the adsorption and surface self-assembly of a cationic singlechain surfactant, dodecyl trimethylammonium bromide $\left(\mathrm{C}_{12} \mathrm{TAB}\right)$, and its related Gemini surfactant dimethylene$\alpha, \beta$ - bis(dodecyldimethylammonium bromide) [12-2$12] \mathrm{Br}_{2}$, on $(5,7)$, and $(10,14)$ SWNTs in aqueous solution at ambient conditions. They found that the morphology of surfactant aggregates on the SWNT is influenced by the surfactant structure. The same number of [12-2-12] $\mathrm{Br}_{2}$ Gemini surfactants adsorbed are able to cover a larger surface area of SWNT and their head-groups were protruded more extensively toward the aqueous phase, preventing water molecules from accessing the nanotube surface. Representative simulation snapshots of [12-6$12 \mathrm{Br}_{2}$ surfactants adsorbed on the nanotube surface are shown in Fig. 3. The snapshot indicates that the two tailgroups of most of the [12-6-12] $\mathrm{Br}_{2}$ molecules are rotated away from the nanotube surface such that either their terminal tail particles or some of tail particles are in direct contact with the SWNT while their hydrophilic headgroups are projected towards the aqueous phase.

The effects of CNT length, diameter, chirality (armchair and zigzag) and surfactant structures on CNT interaction and dispersion in water/surfactant systems were investigated by Farouk et al. [33] via MD simulations for $(5,5)$, $(5,0)$, and $(10,10)$ SWCNTs with two commonly used surfactants [viz., SDS and sodium dodecylbenzene sulfonate (SDBS)] at room conditions. They revealed that CNT length and diameter as well as optimum amount of surfactant addition and its structures can significantly affect CNT interactions. Surfactant molecules were found to adsorb at the CNT surface and reduced interaction strength between CNTs. SDBS surfactant contributed weaker interactions between CNTs as compared with that of SDS surfactant by a factor of about 10 indicating that SDBS is better than SDS for dispersing CNTs in an aqueous suspension.

Recently, Blankschtein et al. [34] have reported the first detailed large-scale all-atomistic MD simulation study of the adsorption and surface self-assembly of a common bile salt surfactant, sodium cholate (SC), on a SWNT in aqueous solution. They found that the cholate ions wrap around the SWNT like a ring and have a small tendency to orient perpendicular to the cylindrical axis of the SWNT, a unique feature that has not been observed for conventional linear surfactants such as SDS. In addition, they found that, at the saturated surface coverages, SC is a better stabilizer than SDS, a finding that is consistent with the widespread use of SC to disperse SWNTs in aqueous media.

The ability of cationic-rich and anionic-rich mixtures of (cetyltrimethylammonium bromide) CTAB and SDS for dispersing of CNTs in aqueous media has been studied by
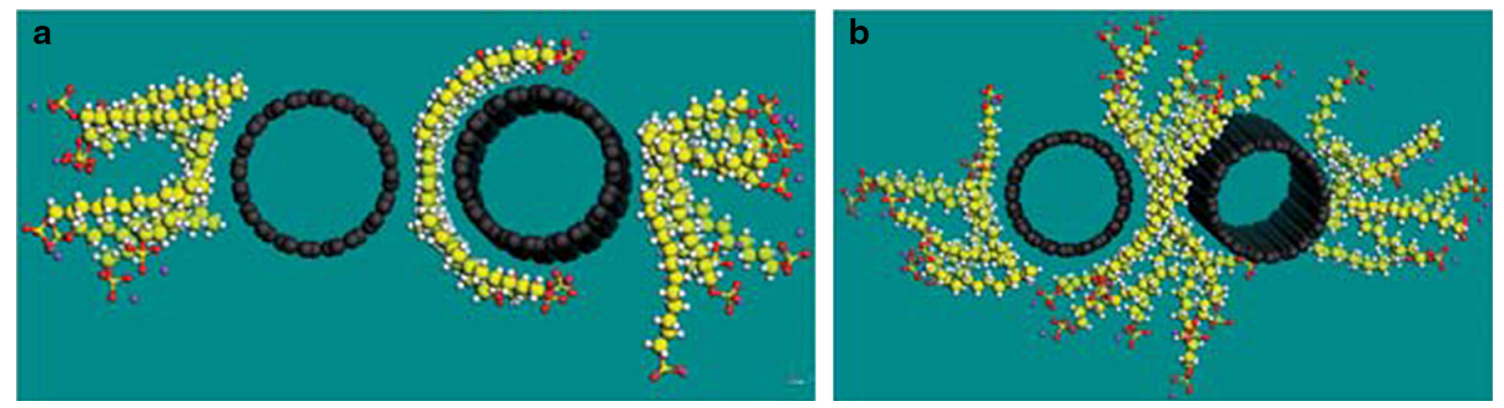

Fig. 2 Separation of two (10, 10) CNTs with a 24 SDS molecules, b 34 SDS molecules. Reproduced (Adapted) from Ref. [31] Copyright (2015), with permission of The Royal Society of Chemistry (RSC) 


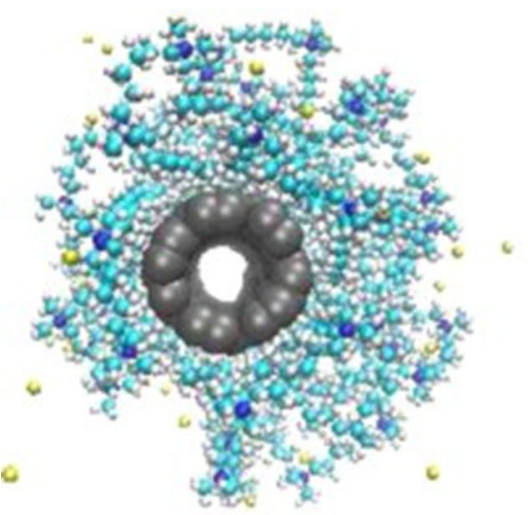

Fig. 3 Representative simulation snapshots of a $(5,7)$ SWNT covered with [12-6-12] $\mathrm{Br}_{2}$, showing the surface structures of the [12-6-12] $\mathrm{Br}_{2}$ at high surface coverage $\left(2.27\right.$ molecule $\left./ \mathrm{nm}^{2}\right)$. The plot on the right is a side view, and the plot on the left is corresponding front view. Water

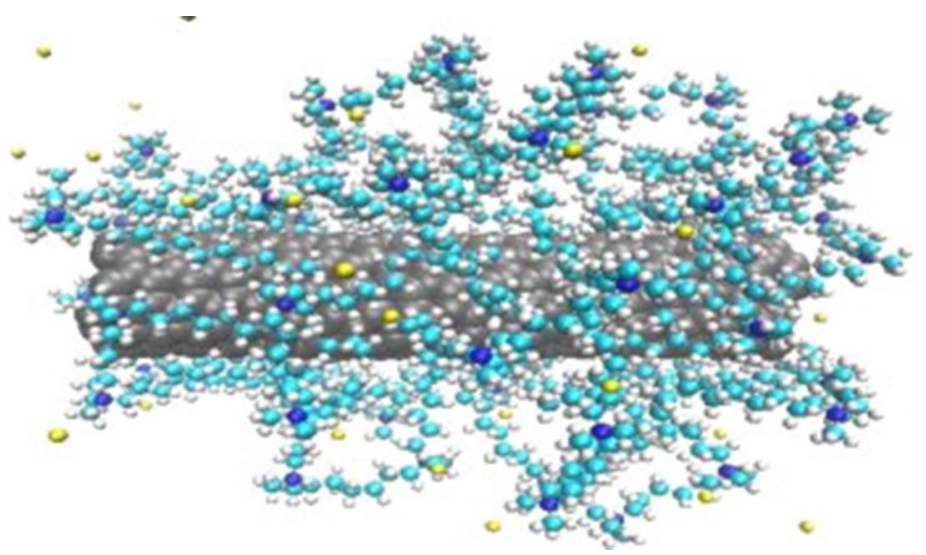

molecules are not shown for clarity. Color code: blue, nitrogen; yellow, bromide counterion; cyan, carbon; white, hydrogen; gray, carbon atoms in the SWNT. Reprinted from Ref. [32] Copyright (2015), with permission from Elsevier

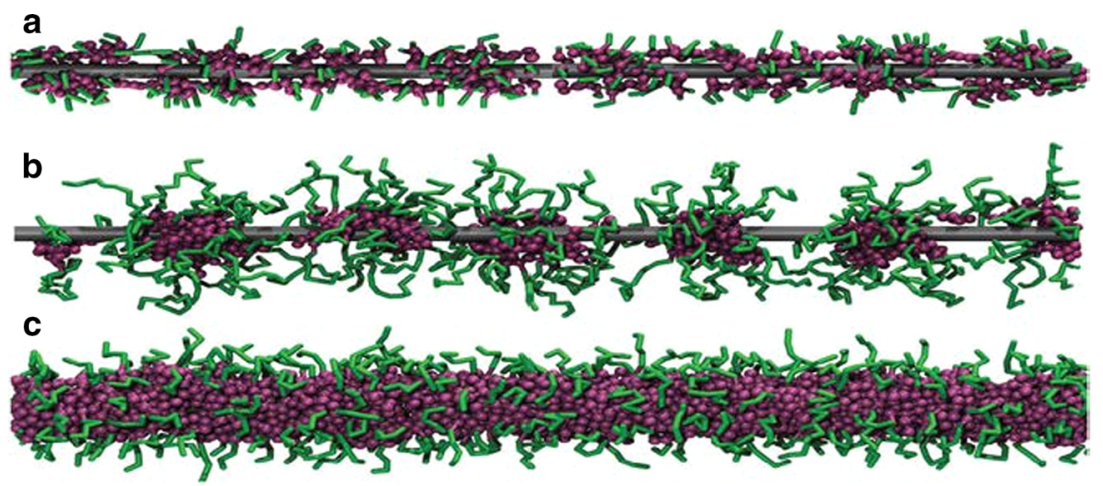

Fig. 4 Snapshots of the three structures amphiphilic molecules can form on CNTs: a Langmuir-type layer, $\mathbf{b}$ adsorbed spherical micelles and $\mathbf{c}$ encapsulation by a cylindrical micelle. Hydrophobic tails are

Sohrabi et al. [35] through the MD simulation method. They found that; compared to the pure CTAB and SDS, these mixtures are more effective with the lower concentrations and more individual CNTs, reflecting a synergistic effect in these mixtures. The synergistic effects observed in mixed surfactant systems are mainly due to the electrostatic attractions between surfactant heads. The results indicated that the hydrophobic interactions between surfactant tails also give rise to the higher adsorption of surfactant molecules. The MD simulation results indicated that the random and disordered adsorption of mixed surfactants onto CNTs may be preferred for a low surfactant concentration. Combining the results from mesoscale and atomistic simulations, Bock et al. [36] have showed that weakly amphiphilic molecules adsorb as Langmuir-type monolayers on CNTs. The molecules adsorb with their hydrophobic parts, thus, creating a uniform structure of cylindrical symmetry where the hydrophilic head-groups preferentially point away from the tubes (Fig. 4a). A shown in magenta and hydrophilic heads in green. Reproduced (Adapted) from Ref. [36] Copyright (2015), with permission of The Royal Society of Chemistry (RSC)

superficially similar structure is formed by strongly adsorbing amphiphilic molecules that tend to form wormlike micelles in bulk solution. As the symmetry of these worm-like micelles matches the symmetry of the tubes, adsorbed molecules self-assemble into a cylindrical micelle that encapsulates the tube (Fig. 4c). The critical difference between encapsulation by a cylindrical micelle and the Langmuir-type monolayer is that the adsorbed micelle forms cooperatively. This stabilizes the adsorbed layer against external perturbations beyond the effect of the adsorption energy. The third structure is formed by strong amphiphilic that prefer higher curvature aggregates and self-assemble into spherical micelles in bulk solution and on the tubes (Fig. 4b).

The effect of surfactant molecular structure on the properties of aqueous surfactant self-assembled aggregates was investigated by Striolo et al. [37] using all-atom MD simulations. To quantify how the surfactant molecular structure affects self-assembly, SDBS surfactants with the 
head-group located either on the fifth or on the twelfth carbon atom along the dodecyl tail were considered. The results suggested that the surfactant molecular structure strongly affects the packing of surfactants on the nanotubes, therefore modulating effective nanotube-nanotube interactions.

$\mathrm{Xu}$ et al. [38] were selected four silicone surfactants (named $\mathrm{S}_{1} \mathrm{E}_{19}, \mathrm{~S}_{2} \mathrm{E}_{38}, \mathrm{~S}_{2} \mathrm{E}_{16}$ and $\mathrm{S}_{1} \mathrm{E}_{16} \mathrm{P}_{8}$ ) were used to disperse CNT in aqueous solutions. The effects of surfactant structure and concentration on the ability at dispersing CNT were considered. All of the four silicone surfactants can disperse CNT in aqueous solution and the sample with $1000 \mathrm{mg} \mathrm{L}^{-1} \mathrm{~S}_{1} \mathrm{E}_{16} \mathrm{P}_{8}$ was the best one. The hydrophilic group polyoxyethylene (PEO) and the hydrophobic group's siloxane and polypropylene (PPO) are crucial factors in the ability of dispersing $\mathrm{CNT}$. $\mathrm{S}_{2} \mathrm{E}_{38}$ with more ethylene oxide (EO) groups has a stronger ability to disperse CNT than $\mathrm{S}_{2} \mathrm{E}_{16}$. The dispersion system provided by $\mathrm{S}_{1} \mathrm{E}_{19}$ which contains fewer siloxane and EO groups was relatively unstable and disperses less CNT. These experimental results have been explained by $\mathrm{MD}$ simulation. $\mathrm{S}_{2} \mathrm{E}_{38}$ compared with $\mathrm{S}_{1} \mathrm{E}_{19}$ and $\mathrm{S}_{2} \mathrm{E}_{16}$ has stronger interactions with CNT. The interaction energy of CNT with $\mathrm{S}_{1} \mathrm{E}_{16} \mathrm{P}_{8}$ which has a PPO moiety, but fewer siloxane groups was close to that of $\mathrm{S}_{2} \mathrm{E}_{16}$.

The dispersion mechanism of aggregated CNTs using Triton X-100 surfactant under various concentrations was investigated by Foroutan et al. [39] with and in without water molecules via MD simulation. The obtained results showed that because of interaction between water molecules and hydrophilic segments of surfactant, water molecules play a significant role in the manner of adsorption of the surfactant on the CNTs surface. In the presence of water molecules, the surfactant molecules do not able to wrap the CNTs and they located in the neighborhood of the CNTs. The results suggested that the creation of space between two CNTs in the absence of the surfactant is performed slowly while in the presence of the surfactant molecules, the creation of space between two CNTs which is leading to the dispersion of the CNTs is remarkable. The surfactant molecules cause that more numbers of water molecules introduce in the vicinity and between the CNTs, and with increasing the radial distances between two CNTs, the number of water molecules is rapidly increased. In addition, they have used the MD simulation to examine the behavior of Triton surfactants in CNTs and bundle [40, 41]. The result of their simulations showed; that the strong intermolecular interaction between CNTs and Triton X-100 that cannot be influenced by the temperature and with an increase in CNTs diameter, the interaction energy was increased accordingly.

Zerbetto el al. [42] have showed that all the manners of surfactant-CNT interactions, cylindrical micelle, hemicelles,

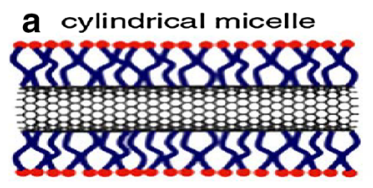

b hemimicelle

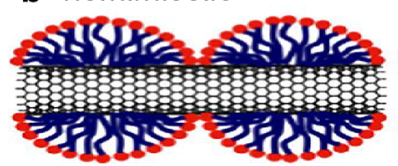

Fig. 5 Schematic illustration of various surfactant assembly structures on a SWNT, including a cylindrical micelles, side and crosssection views, b hemimicelle, and $\mathbf{c}$ random adsorption, Reprinted from Ref. [43] Copyright (2015), with permission from Elsevier

random adsorption are possible and smoothly change one into the other as the concentration or the nature of the surfactant changes. Figure 5 shows various surfactant assembly structures on a SWNT.

Their obtained results show that although the CNT surface is hydrophobic and tends to attract the hydrophobic tail of the surfactants, at low concentration of surfactants the attraction competes with their tendency to form micelles. By spreading evenly on the CNTs, the surfactants would decrease the CNT area in contact with water and they would also increase the area of the tail in contact with water with no net overall free-energy benefit.

\section{Dispersion of CNTs by polymer}

The nature of the dispersion problem for CNTs is rather different from other conventional fillers, such as spherical particles and carbon fibers, because CNTs are characteristic of small diameter in nanometer scale with high aspect ratio $(>1000)$, and thus extremely large surface area. In addition, the commercialized CNTs are supplied in the form of heavily entangled bundles, resulting in inherent difficulties in dispersion. Although many techniques such as density gradient ultracentrifugation, di-electrophoresis, and dispersion by surfactants or polar biopolymers have been developed, so-called conjugated polymer wrapping is one of the most promising and powerful purification and discrimination strategies.

Recently, Loi et al. [44] have studied the debundling and dispersion of SWNTs by wrapping semi-flexible conjugated polymers, such as poly (9,9-dialkylfluorene)s ( $\mathrm{PFx})$ or regioregular poly (3-alkylthiophene)s $\left(\mathrm{P}_{3} \mathrm{AT}\right)$, around the SWNTs, and was accompanied by SWNT discrimination by diameter and chirality. Thereby, the $\pi$-conjugated backbone of the conjugated polymer interacts with the twodimensional, graphene-like $\pi$-electron surface of the nanotubes and the solubilizing alkyl side chains of optimal length support debundling and dispersion in organic 
solvents. They have shown that the alkyl chain length of eight carbons is favored for the dispersion of SWNTs with diameters of $0.8-1.2 \mathrm{~nm}$ and longer alkyls with 12-15 carbons can efficiently interact with nanotubes of increased diameter up to $1.5 \mathrm{~nm}$. They demonstrated that poly $(9,9-$ dialkylfluorene)s with increasing lengths of alkyl chains can interact with nanotubes of larger diameter up to $1.5 \mathrm{~nm}$ (Fig. 6). Therefore, the wrapping and selection mechanism of nanotubes is not only dictated by the nature of the polymer backbone, but also by the length of the alkyl side chains. The chirality map depicted in Fig. 6 illustrates that polyfluorenes with two n-hexyl side chains are mostly ineffective for SWNT dispersion, while PFO wraps only a few varieties of low diameter, chiral SWNTs.

In the similar work, MD simulations of the poly [9,9dioctylfluorenyl-2,7-diyl] (PFO)-SWNT hybrids in toluene were carried out to evaluate the energetics of different wrapping geometries [46]. They showed that the helical wrapping has much lower potential energy because it allows the zipping of the alkyl tails regardless of the tube diameter or chirality. Notice in Fig. 7 that the wrapping geometry corresponding to chains aligned along the nanotube axis in $(8,6)$ SWNT cannot perfectly attach through octyl-octyl contacts due to the relative sizes of alkyl tail and nanotube diameter. They also considered the possibility that groups of three PFO chains wrap around the tubes forming helices, as illustrated in Fig. 7. Differently from previous studies [47], they found helical arrangements in which the conjugated polymer backbone faces the tube wall. One of the octyl chains also wraps around the tubes while the other points toward the solvent.

To explain the solubility of the CNT, wrapped with Chitosan of a $60 \%$ degree of deacetylation (DD), MD simulations were applied by Hannongbua et al. [48] to represent three Chitosan concentrations, using two pristine CNTs (pCNT-pCNT), and one and two CNTs wrapped (pCNT-cwCNT and cwCNT-cwCNT). They indicated the pCNT aggregates due to the hydrophobic and van der Waals interactions between the aromatic rings of the pCNTs. They showed that in the high-concentration Chitosan model (cwCNT-cwCNT) the two cwCNTs were totally separated, freely rotated and well dispersed in the aqueous solution. In addition, Fu et al. [49] were utilized MD simulations to probe the interfacial enhancement between aromatic polymers and SWNT induced by molecular orientation. Two aromatic polymers, polyphenylene sulfide (PPS) and polystyrene (PS) were chosen for comparison. They found that the orientation of polymer chain could bring about an obvious promotion in interfacial interaction for both systems. In PPS/SWNT systems, the increased interfacial interaction energy was due to the easy formation of offset $\pi-\pi$ stacking, while in PS/SWNT systems the formation of edge-to-face $\pi-\pi$ stacking contributed to the enhancement. The mechanism of the orientation induced enhancement was a combination of

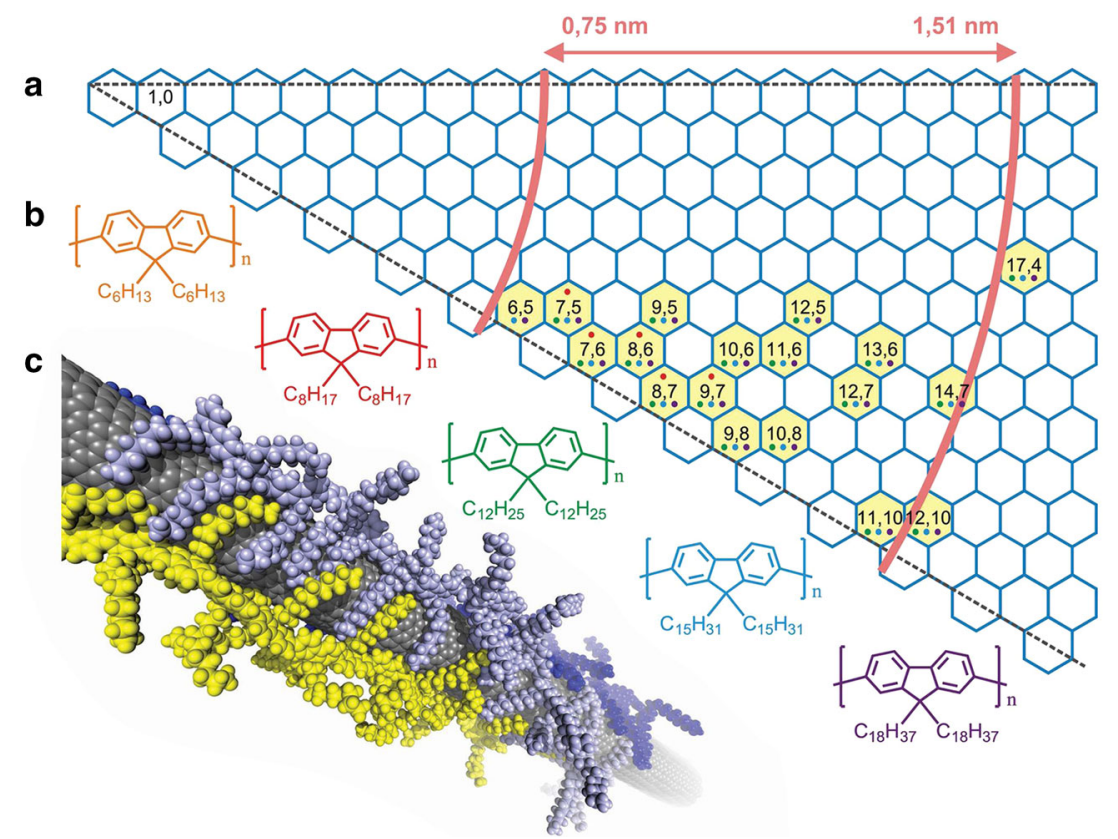

Fig. 6 a Chirality map of polyfluorene-wrapped SWNTs. Selected SWNTs are highlighted in yellow; the color of the dots inside the hexagons represents the polyfluorene derivatives that are able to select the nanotubes (with the color code used for the chemical structures). b Chemical structures of the polyfluorene derivatives tested: $\mathrm{PF}_{6}, \mathrm{PF}_{8}$ (commonly known as $\mathrm{PFO}$ ), $\mathrm{PF}_{12}, \mathrm{PF}_{15}$, and $\mathrm{PF}_{18}$; the numbers indicate the alkyl chain length. $\mathbf{c}$ Structure of SWNT-polymer hybrids as obtained by $\mathrm{MD}$ simulation; the image depicts three $\mathrm{PF}_{12}$ chains wrapped around a $(12,10)$ nanotube, Reprinted from Ref. [45] Copyright (2015), with permission from Wiley-VCH 


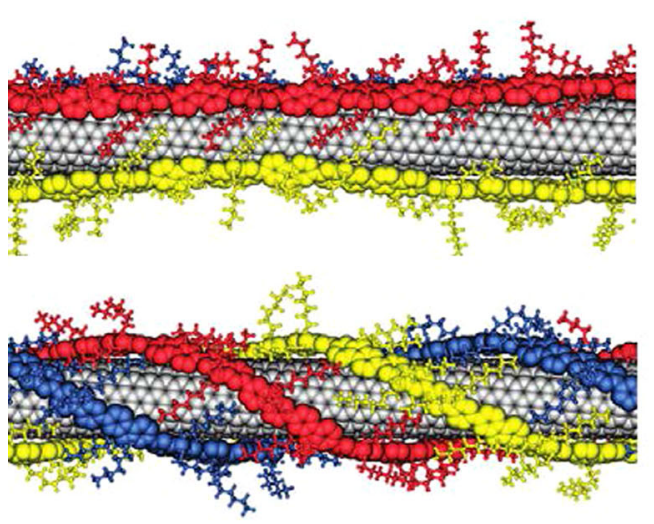

Fig. $7(8,6)$ Nanotube wrapped by three PFO chains (represented as blue, red, and yellow structures) in two geometries: chains aligned to the tube axis (top, left) and forming helices (bottom, left). The solvated system included enough toluene molecules (light gray

forming more $\pi-\pi$ stacking and better coating effect. In this regard, Foroutan el al. [50] have investigated the interfacial binding between the SWNTs and conjugated polymers including polythiophene (PT), polypyrrole (PP), poly (2,6-pyridinylenevinylene-co-2,5-dioctyloxy-p-phenylenevinylene) (PPyPV), and poly(m-phenylenevinyleneco-2,5-dioctyloxy-p-phenylenevinylene) (PmPV) and showed that the intermolecular interaction was strongly influenced by the specific monomer structure of conjugated polymer and nanotube radius.

The influence of the SWNTs' position, the polymer chain length and the temperature on the interaction force between the two neighboring SWNTs are systematically studied by Zhang et al. [51]. They indicated that; (1) The dispersion angle dominates the amplitude and the interaction force evolution, with or without polymer during the pulling process of two SWNTs. (2) The chain length does not affect the two SWNTs' interaction force within a short separation distance, (3) The temperature has a minor influence on the maximum pull force, while the increased temperature greatly decreases the pullout energy. (4) Based on the detailed analysis of the separation process, the selfrepairing function of the system was found. The separation force evolutions of the bare junction (pure binding of two SWNTs) and $30 \mathrm{PE}$ molecules of chain length with 20 monomers are illustrated in Fig. 8 for zero dispersion angle. Multi-peaks and valleys appear in the force-separation curve for the wrapped polymer chains. For two pure SWNTs, the force-separation curve exhibits only one maximum peak. However, the wrapped junction presents a much longer binding range than the bare junction, while the "force enhancing point" locates at $0.38 \mathrm{~nm}$ (the interaction point after the first valley).

Pasquinelli et al. [52] have explored the interface between SWNTs and polymer chains with semi-flexible

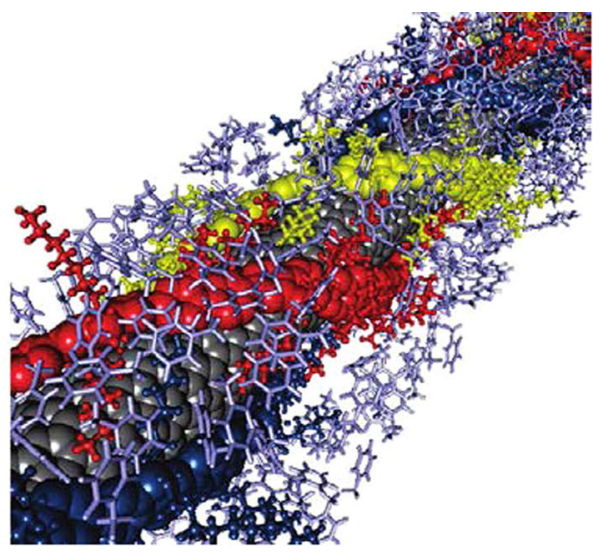

structures) to cover the octyl side chains (right). Reprinted (adapted) with permission from Ref. [46]. Copyright (2015) American Chemical Society

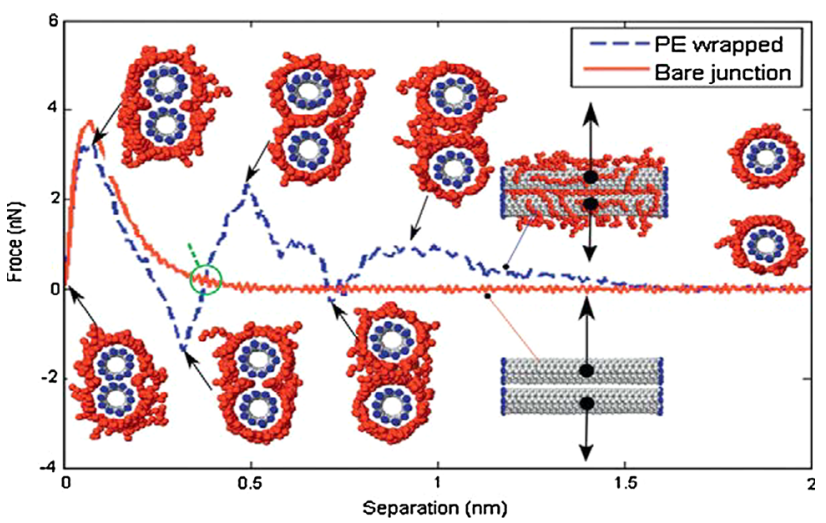

Fig. 8 Separation evolution for double SWNTs at $300 \mathrm{~K}$, Reprinted from Ref. [51] Copyright (2015), with permission from Elsevier

and stiff backbones via MD simulations. These simulations investigate the structural and dynamical features of interactions with the SWNT, such as how the polymers prefer to interface with the SWNT and how the interfacial interaction is affected by the chemical composition and structure of the polymer. Their simulations indicated that polymers with stiff and semi-flexible backbones tend to wrap around the SWNT with more distinct conformations than those with flexible backbones. In addition, Kilina et al. [53] have investigated the morphology and dispersion of a variety of SWNTs non-covalently functionalized by carbazole polymers. Their results elucidate that isomer types of polycarbazoles together with their length govern morphology of carbazole-SWNT hybrids and, hence, their dispersion and bundling properties. The $\pi-\pi$ stacking between the carbazole and the SWNT results in a stable carbazole-SWNT hybrid complex with the SWNT-carbazole interaction increasing with decreasing in the polymer length. The small size of carbazole oligomers and their localization on 
one side of the nanotube prevents tube unbundling. Farouk et al. [54] were carried out MD simulations to investigate CNT interactions and dispersion in a PEO/water solution. Their simulation results provide detailed atomic arrangements and atomic interactions between the CNTs and surrounding molecules (PEO and water). They found that, in the CNT/water system at larger CNT-CNT separations, both the desolvation barrier and the solvent-separated minimum are relatively weak, and the water-mediated force of interaction is attractive distances.

Mayor et al. [55] have reported the unexpected selectivity of poly( $N$-decyl-2,7-carbazole) to almost exclusively disperse semiconducting SWNTs with differences of their chiral indices $(n-m) \geq 2$ in toluene. The observed selectivity complements perfectly the dispersing features of the fluorine analog poly (9,9-dialkyl-2,7-fluorene), which disperses semiconducting SWNTs with $(n-m) \leq 2$ in toluene. All-atom molecular modeling with decamer model compounds of the polymers and $(10,2)$ and $(7,6)$ SWNTs suggests differences in the $\pi-\pi$ stacking interaction as the origin of the selectivity. They observed energetically favored complexes between the $(10,2)$ SWNT and the carbazole decamer and between the $(7,6)$ SWNT and the fluorene decamer, respectively. Fourteen different "hairyrod" conjugated polymers, 9,9-dioctylfluorene derivatives entailing 1,2,3-triazole, azomethine, ethynyle, biphenyle, stilbene, and azobenzene lateral units, are synthesized via modular conjugation and are systematically investigated with respect to their ability to selectively disperse SWNTs by Wenzel et al. [56]. They showed that, four polymers of the azomethine type, with unprecedented selectivity toward dispersing $(8,7),(7,6)$, and $(9,5)$ SWNT species, have been identified. In particular, azomethine polymers, have been evidenced to be very effective in the highly selective solubilization of SWNTs. The experimentally observed selectivity results are unambiguously supported by MD simulations that account for the geometrical properties and deformation energy landscape of the polymer. Specifically, the calculations accurately and with high precision predict the experimentally observed selectivity for the $(7,6)$ and $(9,5)$ conformations. Dispersion of the aggregated nonbundled and bundled CNTs in the 1-n-propyl-4-amino1,2,4-triazolium bromide ionic liquid was recently investigated by Foroutan et al. [57]. They showed that in nonbundled systems, the structure of the aggregated CNTs begins to be separated from the area which has larger contact surface with the solvent. The solvent ions, specially the cations, weaken the $\pi-\pi$ interactions of nanotubes by their shielding effect and producing the $\pi-\pi$ stacking interactions. The temporary resistance of the interior CNTs in this system is due to their larger contact surface with their neighboring nanotube and less contact surface with the surrounding ionic liquids. For the bundled systems, it was found that they severely resist against the solvent separating forces. This happens because in these systems, the sum of $\pi-\pi$ interactions between the common surfaces of the nanotube molecules is larger than these interactions in the corresponding non-bundled systems due to the less contact surface of the solvent with the bundled CNTs. The comparison of the behavior of the bundled systems with the non-bundled ones shows this fact that the stability of the bundled system against separation is more than the similar non-bundled ones. Indeed, what causes the stability and aggregation of nanotubes beside each other is the $\pi-\pi$ attraction interaction between quasi-benzene rings in neighboring nanotube molecules [58].

\section{Dispersion of CNTs by surface functionalization}

The terms 'functionalization' and 'surface modification' have been widely and indiscriminately used to describe the introduction of various types of functional groups onto CNT surfaces, which act as reaction sites for subsequent modifications. With the expansion of research into the CNT surface modification, these terms must be refined to describe the nature of modifications more precisely. Given that 'surface modification' involves pre-treatments to improve dispersions of CNTs, this term must be understood more clearly, particularly in relation to the dispersion behavior of CNTs in a specific surrounding medium. Various covalent functionalization strategies have been reported experimentally: (1) Defect site creation, and functionalization from the defect sites [59], (2) Creation of carboxylic acids on the end caps of carbon nanotubes and subsequent derivatization from the acids [60], (3) Covalent sidewall functionalization [61]. In particular, covalent functionalization of CNTs has been accomplished using three different approaches: thermally activated chemistry, electrochemical modification, and photochemical functionalization [62]. Although covalent functionalization methods have also been developed for MWCNTs [63], fewer investigations have been devoted to this type of nanotube. A number of routes to functionalized CNTs have been reported experimentally, including small molecules [64], linear polystyrene (PS) [65], hyperbranched poly (urea-urethane) [66], biodegradable poly ( $\varepsilon$-caprolactone) (PCL) [67], ionic polymers [68], and photoresponsive polyurethane containing azobenzene [69] are well soluble in water or organic solvents. In this regard, Subramanian et al. [70] were utilized classical MD simulations to investigate the covalent functionalization of CNT and its interaction with ethylene glycol (EG) and water molecules. The MD simulation reveals the dispersion of functionalized CNT and the prevention of aggregation in aqueous medium. Further, they showed that, as the presence of a number 
of functionalized nanotube increases, an enhancement in the propensity for the interaction with water molecules can be observed. They found that the relative enhancement in the interaction of water molecules with functionalized CNT is highly favorable when compared to the interaction of EG.

In addition, the behavior of the $(8,2)$ CNTs and functionalized carbon nanotubes (FCNTs) with four functional groups in water were studied by Foroutan et al. [71]. Glutamine as a long chain functional group and carboxyl as a short chain functional group have been used as functional groups in FCNTs. Four functional groups in each FCNT were localized at two positions: (i) all four functional groups were in the side walls of nanotube, (ii) two functional groups were at the ends and two functional groups were in the side walls of nanotube. They showed that the position of the functional groups in FCNTs has an important role in the interaction of hydrophilic groups of FCNTs with water molecules. Furthermore, they also investigated the behavior of FCNTs with sixteen carboxyl functional groups in water. The presence of these large numbers of carboxyl functional groups on the CNTs prevents water molecules from moving towards hydrophilic carboxyl functional groups. This demonstrates the advantage of using a lower number of functional groups, each containing many hydrophilic groups like glutamine functional group.
A great number of routes to noncovalent modification of carbon nanotubes have been reported. In this way, the original tubular structure is preserved and the technique does not damage the surface of the CNTs. Noncovalent carbon nanotube functionalization strategies can be classified into two groups: (a) via $\pi-\pi$ interactions, or (b) by electrostatic interactions. Since nanotube covalent functionalization may significantly alter the robustness of the system as well as its optical, electrical, and thermal properties, noncovalent nanotube functionalization approaches should be exploited [72]. Functionalization via $\pi-\pi$ interactions relies on the interactions established between CNTs and nonpolar molecules when these molecules contain aromatic rings or $\mathrm{C}=\mathrm{C}$ bonds. These interactions could be very stable [73] and are caused by $\pi-\pi$ stacking. A $\pi-\pi$ interaction is an electrostatic interaction in which the offset and/or orientation of the $\pi$ orbitals on opposing molecules maximize the $\sigma-\pi$ attractive interactions while minimizing the opposing $\pi-\pi$ repulsive interactions. Blankschtein et al. [74] have combined MD simulations, experiments, and equilibrium reaction modeling to both understand and model the extent of diazonium functionalization of SWNTs coated with various surfactants. They showed that the free energy of diazonium adsorption, can be used to rank surfactants in terms of the extent of functionalization attained following their adsorption on the nanotube
Table 1 Summery of the MD simulations of the dispersion of CNTs with different compounds and the details of the simulations

\begin{tabular}{|c|c|c|c|c|}
\hline \multirow[t]{2}{*}{ Compound } & \multirow[t]{2}{*}{ Type of compound } & \multicolumn{2}{|c|}{ Details of the simulations } & \multirow[t]{2}{*}{ Refs. } \\
\hline & & Package & Force field & \\
\hline SDS and SC & Surfactant & Cerius2 & CVFF-950 & [29] \\
\hline FMN & Biomolecule & GROMACS & AMBER & {$[30]$} \\
\hline $\mathrm{C}_{12} \mathrm{TAB}$ and Gemini & Surfactant & GROMACS & OPLS-AA & {$[32]$} \\
\hline SDBS and SDS & Surfactant & NAMD & CHARMM & {$[33]$} \\
\hline $\mathrm{SC}$ & Surfactant & GROMACS & OPLS-AA & {$[34]$} \\
\hline CTAB and SDS & Surfactant & GROMACS & United-atom GROMOS & {$[35]$} \\
\hline Triton X-100 & Surfactant & Tinker & AMBER & [39] \\
\hline PFO & Polymer & Cerius2 & CVFF-950 & [46] \\
\hline Chitosan & Biopolymer & AMBER & GLYCAM & [48] \\
\hline PPS and PS & Polymer & Materials Studio & COMPASS & [49] \\
\hline PE & Polymer & LAMMPS & AIREBO & {$[51]$} \\
\hline PA & Polymer & DL_POLY & DREIDING & {$[52]$} \\
\hline \multicolumn{5}{|l|}{ PET } \\
\hline \multicolumn{5}{|l|}{ PPV } \\
\hline \multicolumn{5}{|l|}{ PPy } \\
\hline \multicolumn{5}{|l|}{ PPE } \\
\hline Carbazole & Polymer & GAUSSIAN & MM3 & {$[53]$} \\
\hline PEO & Polymer & NAMD & CHARMM & {$[54]$} \\
\hline Diazonium & Organic compound & GROMACS & OPLS-AA & {$[70]$} \\
\hline Glutamine & Amino acid & Tinker & AMBER & [71] \\
\hline
\end{tabular}


surface. The difference in binding affinities between linear and rigid surfactants is attributed to the synergistic binding of the diazonium ion to the local "hot/cold spots" formed by the charged surfactant heads. In summarizing the results of the dispersion of CNTs by simulation with different compounds and their dispersion mechanisms and details of the simulations are listed in Table 1.

\section{Conclusions}

Most scientist's research in the field has been directed towards finding a good surfactant/polymer to disperse CNTs. The continuity of this effort underlines its importance and highlights the fact that we have not been sufficiently successful. Although quite some knowledge has been gained in the process, only now it begins to coalesce into understanding. Computer simulations play a key role in this process. In our view, significant progress in the field can only be achieved through understanding. Understanding on the level of a single tube-tube contact should be provided by computer simulations and be verified by experiments. MD simulations provide guidelines to exfoliate, solvate, and stabilize CNTs in solution. They suggest that it is necessary to employ dispersing agents that (1) strongly adsorb on the nanotube surface, (2) present hydrophilic groups, which function better if they are rigid, and (3) are not very mobile on the nanotube surface. Nanotube diameter and chirality sorting can be obtained if the dispersing agents show aggregates with a structure that depends on the nanotube geometry.

Open Access This article is distributed under the terms of the Creative Commons Attribution 4.0 International License (http://creati vecommons.org/licenses/by/4.0/), which permits unrestricted use, distribution, and reproduction in any medium, provided you give appropriate credit to the original author(s) and the source, provide a link to the Creative Commons license, and indicate if changes were made.

\section{References}

1. De Volder, M.F.L., Tawfick, S.H., Baughman, R.H., Hart, A.J.: Carbon nanotubes: present and future commercial applications. Science 339, 535-539 (2013)

2. Miao, M.: Electrical conductivity of pure carbon nanotube yarns. Carbon 49, 3755-3761 (2011)

3. Marconnet, A.M., Panzer, M.A., Goodson, K.E.: Thermal conduction phenomena in carbon nanotubes and related nanostructured materials. Rev. Mod. Phys. 85, 1295-1326 (2013)

4. Bianco, A., Kostarelos, K., Prato, M.: Making carbon nanotubes biocompatible and biodegradable. Chem. Commun. 47, 10182-10188 (2011)

5. Leea, J., Junga, Y., Songa, J., Kimb, J.S., Leeb, G.-W., Jeongb, H.J., Jeonga, Y.: High-performance field emission from a carbon nanotube carpet. Carbon 50, 3889-3896 (2012)

6. Noked, M., Okashy, S., Zimrin, T., Aurbach, D.: Composite carbon nanotube/carbon electrodes for electrical double- layer super capacitors. Angew. Chem. 124, 1600-1603 (2012)

7. Kruss, S., Hilmer, A.J., Zhang, J., Reuel, N.F., Mu, B., Strano, M.S.: Carbon nanotubes as optical biomedical sensors. Adv. Drug Deliv. Rev. 65, 1933-1950 (2013)

8. Rahmanian, S., Thean, K.S., Suraya, A.R., Shazed, M.A., Mohd Salleh, M.A., Yusoff, H.M.: Carbon and glass hierarchical fibers: influence of carbon nanotubes on tensile, flexural and impact properties of short fiber reinforced composites. Mater. Des. 43, 10-16 (2013)

9. Heister, E., Neves, V., Lamprecht, C., Silva, S.R.P., Coley, H.M., McFadden, J.: Drug loading, dispersion stability, and therapeutic efficacy in targeted drug delivery with carbon nanotubes. Carbon 50, 622-632 (2012)

10. Zhou, F., Wu, S., Song, S., Chen, W.R., Resasco, D.E., Xinga, D.: Antitumor immunologically modified carbon nanotubes for photothermal therapy. Biomaterials 33, 3235-3242 (2012)

11. Liu, Z., Robinson, J.T., Tabakman, S.M., Yang, K., Dai, H.: Carbon materials for drug delivery and cancer therapy. Mater. Today 14, 316-323 (2011)

12. Franklin, A.D., Luisier, M., Han, S.-J., Tulevski, G., Breslin, C.M., Gignac, L., Lundstrom, M.S., Haensch, W.: Sub-10 nm carbon nanotube transistor. Nano Lett. 12, 758-762 (2012)

13. Tune, D.D., Flavel, B.S., Krupke, R., Shapter, J.G.: Carbon nanotube-silicon solar cells. Adv. Energy Mater. 2, 1043-1055 (2012)

14. Rodrigues, E.G., Carabineiro, S.A.C., Delgado, J.J., Chen, X., Pereira, M.F.R., Órfão, J.J.M.: Gold supported on carbon nanotubes for the selective oxidation of glycerol. J. Catal. 285, 83-91 (2012)

15. Oriňáková, R., Oriňák, A.: Recent applications of carbon nanotubes in hydrogen production and storage. Fuel 90, 3123-3140 (2011)

16. Sanip, S.M., Ismail, A.F., Goh, P.S., Soga, T., Tanemura, M., Yasuhiko, H.: Gas separation properties of functionalized carbon nanotubes mixed matrix membranes. Sep. Purif. Technol. 78, 208-213 (2011)

17. De las Casas, C., Li, W.: A review of application of carbon nanotubes for lithium ion battery anode material. J. Power Sources 208, 74-85 (2012)

18. Herrera-Herrera, A.V., González-Curbelo, M.Á., HernándezBorges, J., Rodríguez-Delgado, M.Á.: Carbon nanotubes applications in separation science: a review. Anal. Chim. Acta 734, 1-30 (2012)

19. Lota, G., Fic, K., Frackowiak, E.: Carbon nanotubes and their composites in electrochemical applications. Energy Environ. Sci. 4, 1592-1605 (2011)

20. Lu, K.L., Lago, R.M., Chen, Y.K., Green, M.L.H., Harris, P.J.F., Tsang, S.C.: Mechanical damage of carbon nanotubes by ultrasound. Carbon 34, 814-816 (1996)

21. Prevoteau, A., Soulié-Ziakovic, C., Leibler, L.: Universally dispersible carbon nanotubes. J. Am. Chem. Soc. 134, 19961-19964 (2012)

22. Kim, S.W., Kim, T., Kim, Y.S., Choi, H.S., Lim, H.J., Yang, S.J., Park, C.R.: Surface modifications for the effective dispersion of carbon nanotubes in solvents and polymers. Carbon 50, 3-33 (2012)

23. Li, Y., Yang, D., Adronov, A., Gao, Y., Luo, X., Li, H.: Covalent functionalization of single-walled carbon nanotubes with thermoresponsive core cross-linked polymeric micelles. Macromolecules 45, 4698-4706 (2012)

24. Tuncel, D.: Non-covalent interactions between carbon nanotubes and conjugated polymers. Nanoscale 3, 3545-3554 (2011)

25. Jiang, C., Saha, A., Xiang, C., Young, C.C., Tour, J.M., Pasquali, M., Martí, A.A.: Increased solubility, liquid-crystalline phase, and selective functionalization of single-walled carbon nanotube polyelectrolyte dispersions. ACS Nano 7, 4503-4510 (2013) 
26. Zhao, Y.-L., Stoddart, J.F.: Noncovalent functionalization of single-walled carbon nanotubes. Acc. Chem. Res. 42, 1161-1171 (2009)

27. Calvaresi, M., Hoefinger, S., Zerbetto, F.: Probing the structure of lysozyme-carbon-nanotube hybrids with molecular dynamics. Chem. Eur. J. 18, 4308-4313 (2012)

28. Tkalya, E.E., Ghislandi, M., De With, M.G., Koning, C.E.: The use of surfactants for dispersing carbon nanotubes and graphene to make conductive nanocomposites. Curr. Opin. Colloid Interface Sci. 17, 225-232 (2012)

29. Carvalho, E.J.F., dos Santos, M.C.: Role of surfactants in carbon nanotubes density gradient separation. ACS Nano 4, 765-770 (2010)

30. Tummala, N.R., Morrow, B.H., Resasco, D.E., Striolo, A.: Stabilization of aqueous carbon nanotube dispersions using surfactants: insights from molecular dynamics simulations. ACS Nano 4, 7193-7204 (2010)

31. Duan, W.H., Wang, Q., Collinsa, F.: Dispersion of carbon nanotubes with SDS surfactants: a study from a binding energy perspective. Chem. Sci. 2, 1407-1413 (2011)

32. Poorgholami-Bejarpasi, N., Sohrabi, B.: Role of surfactant structure in aqueous dispersions of carbon nanotubes. Fluid Phase Equilib. 394, 19-28 (2015)

33. Uddin, N.M., Capaldi, F.M., Farouk, B.: Molecular dynamics simulations of carbon nanotube dispersions in water: effects of nanotube length, diameter, chirality and surfactant structures. Comput. Mater. Sci. 53, 133-144 (2012)

34. Lin, S., Blankschtein, D.: Role of the bile salt surfactant sodium cholate in enhancing the aqueous dispersion stability of singlewalled carbon nanotubes: a molecular dynamics simulation study. J. Phys. Chem. B 114, 15616-15625 (2010)

35. Sohrabi, B., Poorgholami-Bejarpasi, N., Nayeri, N.: Dispersion of carbon nanotubes using mixed surfactants: experimental and molecular dynamics simulation studies. J. Phys. Chem. B 118, 3094-3103 (2014)

36. Angelikopoulos, P., Bock, H.: The science of dispersing carbon nanotubes with surfactants. Phys. Chem. Chem. Phys. 14, 9546-9557 (2012)

37. Suttipong, M., Tummala, N.R., Kitiyanan, B., Striolo, A.: Role of surfactant molecular structure on self-assembly: aqueous SDBS on carbon nanotubes. J. Phys. Chem. C 115, 17286-17296 (2011)

38. Xin, X., Pang, J., Li, W., Wang, Y., Yuan, J., Xu, G.: Dispersing carbon nanotubes in aqueous solutions of trisiloxane-based surfactants modified by ethoxy and propoxy groups. J. Surfactants Deterg. 18, 163-170 (2015)

39. Fatemi, S.M., Foroutan, M.: Study of dispersion of carbon nanotubes by triton X-100 surfactant using molecular dynamic simulation. J. Iran. Chem. Soc. 12, 1905-1913 (2015)

40. Fatemi, Sh, Foroutan, M.: Molecular dynamics simulations studies of triton surfactant-wrapped single-walled carbon nanotubes surface. J. Adv. Phys. (2016). doi:10.1166/jap.2016.1251

41. Fatemi, S.M., Foroutan, M.: Structure and dynamics of a nonionic surfactant within a carbon nanotube bundle by molecular dynamics simulation. J. Colloid Sci. Biotech. 2, 41-45 (2013)

42. Calvaresi, M., Dallavalle, M., Zerbetto, F.: Wrapping nanotubes with micelles, hemimicelles, and cylindrical micelles. Small 5, 2191-2198 (2009)

43. Wang, H.: Dispersing carbon nanotubes using surfactants. Curr. Opin. Colloid Interface Sci. 14, 364-371 (2009)

44. Samanta, S.K., Fritsch, M., Scherf, U., Gomulya, W., Bisri, S.Z., Loi, M.A.: Conjugated polymer-assisted dispersion of single-wall carbon nanotubes: the power of polymer wrapping. Acc. Chem. Res. 47, 2446-2456 (2014)

45. Gomulya, W., Costanzo, G.D., De Carvalho, E.J.F., Bisri, S.Z., Derenskyi, V., Fritsch, M., Fröhlich, N., Allard, S., Gordiichuk, P., Herrmann, A., Marrink, S.J., dos Santos, M.C., Scherf, U.,
Loi, M.A.: Semiconducting single-walled carbon nanotubes on demand by polymer wrapping. Adv. Mater. 25, 2948-2956 (2013)

46. Gao, J., De Loi, M.A., Carvalho, E.J.F., dos Santos, M.C.: Selective wrapping and supramolecular structures of polyfluorene-carbon nanotube hybrids. ACS Nano 5, 3993-3999 (2011)

47. Nish, A., Hwang, J.Y., Doig, J., Nicholas, R.J.: Highly selective dispersion of single-walled carbon nanotubes using aromatic polymers. Nat. Nanotechnol. 2, 640-646 (2007)

48. Rungrotmongkol, T., Arsawang, U., Iamsamai, C., Vongachariya, A., Dubas, S.T., Ruktanonchai, U., Soottitantawat, A., Hannongbua, S.: Increased dispersion and solubility of carbon nanotubes noncovalently modified by the polysaccharide biopolymer, chitosan: MD simulations. Chem. Phys. Lett. 507, 134-137 (2011)

49. Yu, B., Fu, S., Wua, Z., Bai, H., Ning, N., Fu, Q.: Molecular dynamics simulations of orientation induced interfacial enhancement between single walled carbon nanotube and aromatic polymers chains. Compos. Part A 73, 155-165 (2015)

50. Foroutan, M., Nasrabadi, A.T.: Investigation of the interfacial binding between single-walled carbon nanotubes and heterocyclic conjugated polymers. J. Phys. Chem. B 114, 5320-5326 (2010)

51. Zhang, Y., Zhao, J., Wei, N., Jiang, J., Gong, Y., Rabczuk, T.: Effects of the dispersion of polymer wrapped two neighbouring single walled carbon nanotubes (SWNTs) on nanoengineering load transfer. Compos. Part B 45, 1714-1721 (2013)

52. Tallury, S.S., Pasquinelli, M.A.: Molecular Dynamics Simulations of Polymers with Stiff Backbones Interacting with SingleWalled Carbon Nanotubes. J. Phys. Chem. B 114, 9349-9355 (2010)

53. Mayo, M.L., Hogle, D., Yilmaz, B., Köse, M.E., Kilina, S.: Morphology and dispersion of polycarbazole wrapped carbon nanotubes. RSC Adv. 3, 20492-20502 (2013)

54. Uddin, N.M., Capaldi, F.M., Farouk, B.: Molecular dynamics simulations of the interactions and dispersion of carbon nanotubes in polyethylene oxide/water systems. Polymer 52, 288-296 (2011)

55. Lemasson, F.A., Strunk, T., Gerstel, P., Hennrich, F., Lebedkin, S., Barner-Kowollik, C., Wenzel, W., Kappes, M.M., Mayor, M.: Selective dispersion of single-walled carbon nanotubes with specific chiral indices by Poly( $N$-decyl-2,7-carbazole). J. Am. Chem. Soc. 133, 652-655 (2011)

56. Gerstel, P., Klumpp, S., Hennrich, F., Poschlad, A., Meded, V., Blasco, E., Wenzel, W., Kappes, M.M., Barner-Kowollik, C.: Highly selective dispersion of single-walled carbon nanotubes via polymer wrapping: a combinatorial study via modular conjugation. ACS Macro Lett. 3, 10-15 (2014)

57. Mohammadi, M., Foroutan, M.: Mixture of ionic liquid and carbon nanotubes: comparative studies of the structural characteristics and dispersion of the aggregated non-bundled and bundled carbon nanotubes. Phys. Chem. Chem. Phys. 15, 2482-2494 (2013)

58. Fatemi, S.M., Foroutan, M.: Recent findings about ionic liquids mixtures obtained by molecular dynamics simulation. J. Nanostruct. Chem. 5, 243-253 (2015)

59. Fu, K., Huang, W., Lin, Y., Riddle, L.A., Carroll, D.L., Sun, Y.P.: Defunctionalization of functionalized carbon nanotubes. Nano Lett. 1, 439-441 (2001)

60. Hamon, M.A., Hui, H., Bhowmik, P., Itkis, H.M.E., Haddon, R.C.: Ester-functionalized soluble single-walled carbon nanotubes. Appl. Phys. A 74, 333-338 (2002)

61. Yu, M.-F., Files, B.S., Arepalli, S., Ruoff, R.S.: Tensile loading of ropes of single wall carbon nanotubes and their mechanical properties. Phys. Rev. Lett. 84, 5552-5555 (2000)

62. Balasubramanian, K., Burghard, M.: Chemically functionalized carbon nanotubes. Small 1, 180-192 (2005) 
63. Sainsbury, T., Fitzmaurice, D.: Templated assembly of semiconductor and insulator nanoparticles at the surface of covalently modified multiwalled carbon nanotubes. Chem. Mater. 16, 3780-3790 (2004)

64. Yang, Y.K., Qiu, S.Q., Xie, X.L., Wanga, X., Lib, R.K.Y.: A facile, green, and tunable method to functionalize carbon nanotubes with water-soluble azo initiators by one-step free radical addition. Appl. Surf. Sci. 256, 3286-3292 (2010)

65. Yang, Y.K., Xie, X.L., Wu, J.G., Mai, Y.-W.: Synthesis and selfassembly of polystyrene-grafted multiwalled carbon nanotubes with a hairy-rod nanostructure. J. Polym. Sci. Polym. Chem. 44, 3869-3881 (2006)

66. Yang, Y.K., Xie, X.L., Yang, Z.F., Wang, X., Cui, W., Yang, J., Mai, Y.-W.: Controlled synthesis and novel solution rheology of hyperbranched poly(urea-urethane)-functionalized multiwalled carbon nanotubes. Macromolecules 40, 5858-5867 (2007)

67. Yang, Y.K., Tsui, C.P., Tang, C.Y., Qiu, S., Zhao, Q., Cheng, X., Sun, Z., Li, R.K.Y., Xie, X.: Functionalization of carbon nanotubes with biodegradable supramolecular polypseudorotaxanes from grafted-poly( $\varepsilon$-caprolactone) and $\alpha$-cyclodextrins. Eur. Polym. J. 46, 145-155 (2010)

68. Du, F.B., Tang, C.Y., Xie, X.L., Zhou, X.-P., Tan, L.: Carbon nanotube enhanced gripping in polymer-based actuators. J. Phys. Chem. C 113, 7223-7226 (2009)
69. Yang, Y.K., Wang, X.T., Liu, L., Xie, X., Yang, Z., Li, R.K.Y., Mai, Y.-W.: Structures and photoresponsive behaviors of multiwalled carbon nanotubes grafted by polyurethanes containing azobenzene side-chains. J. Phys. Chem. C 111, 11231-11239 (2007)

70. Balamurugan, K., Baskar, P., Kumar, R.M., Das, S., Subramanian, V.: Effect of functionalization of carbon nanotube on their dispersion in ethylene glycol-water binary mixture-a molecular dynamics and ONIOM investigation. Phys. Chem. Chem. Phys. 16, 24509-24518 (2014)

71. Foroutan, M., Moshari, M.: Molecular dynamics simulations of functionalized carbon nanotubes in water: effects of type and position of functional groups. Physica E 43, 359-365 (2010)

72. Peng, S., Cho, K.: Chemical control of nanotube electronics. Nanotechnology 11, 57-60 (2000)

73. Hirsch, A.: Functionalization of single-walled carbon nanotubes. Angew. Chem. Int. Ed. 41, 1853-1859 (2002)

74. Lin, S., Hilmer, A.J., Mendenhall, J.D., Strano, M.S., Blankschtein, D.: Molecular perspective on diazonium adsorption for controllable functionalization of single-walled carbon nanotubes in aqueous surfactant solutions. J. Am. Chem. Soc. 134, 8194-8204 (2012) 